\title{
Crude Oil Risk Forecasting: New Evidence from Multiscale Analysis Approach
}

\author{
Kaijian He ${ }^{\mathrm{a}, \mathrm{b}}$, Geoffrey K.F. Tso ${ }^{\mathrm{c}}$, Yingchao Zou ${ }^{\mathrm{d}}$, Jia Liu',e \\ ${ }^{a}$ Hunan Engineering Research Center for Industrial Big Data and Intelligent Decision \\ Making, Hunan University of Science and Technology, Xiangtan 411201, China \\ ${ }^{b}$ School of Business, Hunan University of Science and Technology, Xiangtan, 411201, China \\ ${ }^{c}$ Department of Management Sciences, City University of Hong Kong, Tat Chee Avenue, \\ Kowloon Tong, Hong Kong \\ ${ }^{d}$ College of Information Science and Technology, Beijing University of Chemical \\ Technology, Beijing 100029, China \\ ${ }^{e}$ Salford Business School, University of Salford, Greater Manchest, UK
}

\begin{abstract}
Fluctuations in the crude oil price allied to risk have increased significantly over the last decade frequently varying at different risk levels. Although existing models partially predict such variations, so far, they have been unable to predict oil prices accurately in this highly volatile market. The development of an effective, predictive model has therefore become a prime objective of research in this field. Our approach, albeit based in part on previous research, develops an original methodology, in that we have created a risk forecasting model with the ability to predict oil price fluctuations caused by changes in both fundamental and transient risk factors. We achieve this by disintegrating the multi-scale risk-structure of the crude oil market using Variational Mode Decomposition. Normal and transient risk factors are then extracted from the crude oil price using Variational Mode Decomposition and modelled separately using the Quantile Regression Neural Network (QRNN) model. Both risk factors are integrated and ensembled to produce the risk estimates. We then apply our proposed risk forecasting model to predicting future downside risk level in
\end{abstract}

\footnotetext{
*Corresponding author. Tel.:0044-01612952981

Email addresses: kaijian.he@my.cityu.edu.hk (Kaijian He), msgtso@cityu.edu.hk (Geoffrey K.F. Tso), evangeline1203@outlook.com (Yingchao Zou), J.Liu@salford.ac.uk (Jia Liu)
} 
three major crude oil markets, namely the West Taxes Intermediate (WTI), the Brent Market, and the OPEC market. The results demonstrate that our model has the ability to capture downside risk estimates with significantly improved precision, thus reducing estimation errors and increasing forecasting reliability. Key words: Crude oil risk forecasting, Variational Mode Decomposition, Value at Risk, Normal Risk, Transient Risk, Multiscale Analysis, Quantile Regression Neural Network model

\section{Introduction}

A significant body of contemporary research shows that the worldwide crude oil market has been experiencing increasing volatility and risk (Ji and Zhang, 2018; Herrera et al., 2018; Plourde and Watkins, 1998). Many empirical studies demonstrate that movements in prices are driven by recurrent price bubbles and structural breaks such as financial crises and regime changes. For example, Liu and Lee (2018) and Su et al. (2017) identify eight and six such bubbles, respectively, emanating from energy markets. Ji et al. (2018c) find the structural break points for the spillover effect between oil and exchange markets. Ji et al. (2018d) find different behaviour of spillover effect between oil and agricultural commodity markets under bearish and bullish regimes. Balcilar et al. (2014) identify four bubbles and a crisis regime in the WTI and Brent markets. It is arguable that extreme fluctuations are caused by diverse market shocks, unexpected sector innovations such as the rapid development of transportation technology, the internet, unforeseen extreme weather conditions, the development of mining technology, aggressive exploration and the availability of new alternative renewable energy sources, as well as global financial crises and integration with other financial markets (Plourde and Watkins, 1998; Miao et al., 2017; Zhang and Broadstock, 2018; Huang et al., 2017).

Although such (disruptive) factors have exerted an increasingly powerful influence on the crude oil market (Miao et al., 2017; Mensi et al., 2017), until now the ability to measure their effect on future prices has been limited, with a 
lack of predictive tools to inform investors. Before the year 2000, for example, the equilibrium model, which models the supply and demand characteristics of the crude oil price, was accepted as the norm (Huang et al., 2017). Nevertheless, empirical evidence has increasingly suggested that the information linkage and spillover effects between oil and other financial markets such as the stock markets, Carbon market and energy market have been on the rise (Ji et al., 2018b; Zhang, 2017). Financial characteristics due to the increasing financialization of the energy market have become more prevalent in the crude oil market in recent years (Zhang et al., 2018). The influence and impact of component factors driving changes in risk and price cannot be measured accurately by traditional symmetric summary measures such as the standard deviation that focuses on both upside and downside return fluctuations. Subsequently, risk measures placed more emphasis on the potential size of loss and the probability of downside return. Among different risk measures, Value at Risk has become one of the most frequently adopted risk measures, because it has the ability to model the worst expected losses under normal market conditions over the given time horizon (Dowd, 2005). Some use Exponential Weighted Moving Average (EWMA) and GARCH models to capture conditional variance and conditional volatility (Mohammadi and $\mathrm{Su}, 2010$ ). Others use different GARCH models, such as EGARCH and GJR-GARCH, to forecast crude oil variance, and find that the covariance stationary GARCH model performs the best. Wang et al. (2011) show that GARCH-type models are able to capture the long memory effect for crude oil data, which is consistent with the dominant Efficient Market Hypothesis (EMH). These models, however, are built on an equilibrium condition for a stable market environment, and assume homogeneity of market structure, and they are therefore appropriate for stable and stationary market conditions only (Mandelbrot, 1963; Fama, 1965, 1970). In an era beset with unexpected turbulence, they systematically underestimate the market risk consequent upon transient and extreme events, such as the recent global financial crisis. Further advancement includes the Quantile Regression Neural Network (QRNN), which uses Neural Network model to learn the nonlinear relationship 
through quantile variables and explanatory variables. It takes a non-parametric data-driven approach to model risk changes (Koenker and Bassett, 1978; Engle and Manganelli, 2004). The neural network model in QRNN has greater flexibility to model a diverse range of empirical distributions, as well as different, nonlinear relationships between the VaR and the independent variables (Taylor, 2010; Cannon, 2012). Xu et al. (2016) conduct a comprehensive evaluation of the performance of the Quantile Autoregression Neural network (QARNN) model for US, UK and Hong Kong stock markets, providing evidence that QANN demonstrates improved risk estimate accuracy. But QRNN is built on the neural network model and does not reveal much of the underlying risk structure. Researchers have been searching for better risk measures that can incorporate downside risk exposure under extreme conditions.

An alternative approach is to relax the assumption of homogeneity in the EMH and adopt the alternative Fractal Market Hypothesis (FMH), which introduces the multiscale view to account for the distinction between normal and transient risk factors in risk modelling. Multiscale modelling has been well defined in the engineering fields for analysing the complex system that contains sub-systems with characterizing features across scales of time and space (Hosseini and Shah, 2009). In the economics and finance field, the economic and financial system typically demonstrates nonlinear complex behavior, which is characterized by a series of influencing factors differentiated by the scale. These influencing factors can be modelled by a series of models dedicated individually to their multiscale data characteristics. Thus, multiscale models can contribute to the understanding and modeling of the influential factors and multiscale dynamics in the economic and financial system. As many such economic research involves time scale considerations, such as different economic behaviours, over long-term and short-term time horizon, multiscale algorithms have been increasingly adopted in the field to deal with multi-scale issues. Among different multi-scale models, wavelet analysis and Empirical Mode Decomposition (EMD) have recently been increasingly applied to forecast price movements in financial markets. These models can identify influencing factors through extracting sub- 
data series distinguished by scales. These sub-data series are usually simpler and better behaved compared to the original data. They can be modelled more accurately using econometric models. Models based on wavelet and EMD methods produce more accurate forecasts as a consequence (Wang and Wang, 2017; Qiu et al., 2017; Zhang et al., 2018; Yang et al., 2017). For example, Wang et al. (2018a,b) apply the bivariate EMD model to analyse and forecast the crude oil price movement, and show that the addition of wavelet analysis, EMD and VMD models can improve the forecasting accuracy of the mainstream crude oil price forecasting model. Zhu et al. (2017) apply the EMD model to analyse and forecast the carbon market. Wavelet analysis, however, has little flexibility to account for the diverse range of data features found in the crude oil market. Empirical Mode Decomposition takes a quantitative approach to derive the filtering basis from data. Without the theoretical foundation, the number of decomposed components may change significantly over different time periods, causing a mode-mixing problem during the decomposition process. The decomposed components may not correspond to economically meaningful risk factors in the markets.

A new approach, embodying the Variational Mode Decomposition model, is the latest improvement upon the EMD model, searching for constituent modes using the non-recursive optimization-based model based on financial data for forecasting (Dragomiretskiy and Zosso, 2014). For example, Lahmiri (2016) constructs the forecasting model, with hybridization of the VMD model with the Particle Swarm Optimized Neural Network model for six stock markets with improved forecasting accuracy. Jianwei et al. (2017) combine VMD, Independent Component Analysis and ARMA to forecast the crude oil price movement with enhanced accuracy.

In spite of the advances in technical modelling discussed above, none of these approaches has seriously considered the multiscale characteristics of risk estimates in the crude oil forecasting modelling, in spite of wide observations that price fluctuations in the crude oil market are fundamentally determined by the underlying risk factors characterized by their multiscale structure. In our 
paper, we propose a new multiscale approach, incorporating the downside risk level in the crude oil market into the model for forecasting crude oil prices. Our method utilizes the new multi-scale signal processing technique, namely Variational Mode Decomposition, to decompose the underlying risk factors. The deterministic mechanism for each risk factor is modelled using QRNN. Specifically, we search for the multiscale structure of risks and derive the optimal value by using the VMD and QRNN models. We establish that the VMD model extracts data components, or so-called modes in the model. We determine the existence of normal and transient risk factors with their own multiscale characteristics such as fluctuation ranges across ten scales. We use the VaR exceedances (VaR greater than the actual loss) as the criteria. The data series with the lowest number of VaR exceedances is identified as the normal factor (the most conservative risk estimate). The data series with higher VaR exceedances is identified as the transient factor (more aggressive risk estimate). For each extracted risk factor, the QRNN model is estimated with a unique set of parameters. To evaluate its performance, we apply our model to real crude oil price data based on the major crude oil markets. The evaluation results demonstrate that our proposed models produce risk forecasts with improved forecasting accuracy, as compared to the benchmarking model, the Quantile Regression Neural Network model.

Our model makes several significant contributions to the literature. The major contribution is that we demonstrate the existence of both normal and transient risk factors in the crude oil price, which can be captured with more precision by the QRNN model. Recent empirical studies show that crude oil has distinctive behavioural patterns across time scales (Ji et al., 2018a; Huang et al., 2017). We take a step further to show that the selected transient scale behaves significantly differently from the normal factor. The transient scale contains both temporal and extreme factors. Thus, our proposed model is unique in its capability to model these risk factors separately in the transformed multi-scale domain. Our VMD model can separate transient risk factors from normal risk factors and reintegrate these risk factors into forecasting by analysing real data 
characteristics, so as to improve the accuracy of forecasting. Further, we show that although each crude oil price can be decomposed into multiscale data series as the risk factors, the exact scale for both normal and transient risk factors need to be selected based on some criteria. The criteria we choose is the risk forecasts reliability at each scale. Typical performance measures for VaR are the number of VaR exceedances, i.e. the number of times when the actual loss is greater than VaR. We have proposed an empirical method that has an ability to determine the optimal risk decomposition structure more efficiently. We argue that this is of critical importance when we select scales for both normal and transient factors, given that there are many valid candidate scales. The resulting candidate risk structure critically affects the accuracy of forecasting based on multi-scale analysis. The risk structure also critically affects the derivation and generalization of economic and financial theory such as the risk spillover effect and risk measurement. The determination of the optimal risk structure using the multi-scale analysis can improve the robustness of crude oil forecasting, creating an effective tool for informing investors. Overall, work in this paper contributes to the conceptual development in Energy Finance by proposing a new risk forecasting model in the energy risk measurement field. Reliable energy risk measurement is the critical part of the energy risk management system, which is one of the major subjects in the rapid developing Energy Finance discipline (Zhang, 2018; Narayan, 2017; Sadeghi and Shavvalpour, 2006). Despite the increasing adoption of risk measurement models such as Value at Risk to measure downside risk in the energy markets, we have limited knowledge of the underlying risk factors and limited studies on the quantitative risk measurement models have been conducted in recent years (Ji et al., 2018c,d; Narayan, 2017). Our model provides promising modelling approaches to better analyze the risk factors and measure the risk exposure in the energy markets.

The rest of the paper is organized as follows. In Section 2, we discuss wellused models, such as VMD and QRNN, and develop our proposed model. In Section 3, we apply our proposed model to real crude oil data. Section 4 summarises our main findings and provides concluding remarks. 


\section{Modelling and hypothesis development}

\subsection{Variational Mode Decomposition model and Quantile Regression Neural} Network model

Empirical mode decomposition has gained increasing attention and has inspired a series of researches (Huang et al., 1998). The method is data driven and adaptive, i.e. the parameter in the algorithm changes automatically when it is applied to a different set of empirical data. It can extract data features from a mixture of diverse ranges of data. The EMD model is often confronted by the mode mixing problem, when the extracted modes contain components characterized by more than one frequency. The extracted modes are thus not uniquely defined in terms of a fluctuation boundary because this may overlap the extracted modes. VMD is a recently developed new method to solve the mode mixing problem in this line of research (Dragomiretskiy and Zosso, 2014). It decomposes the time series into a finite number of data components, known as modes, using a non-recursive optimization based approach. Given the original crude oil data and the number of modes to decompose, the VMD model produces the exact number of modes required, with limited bandwidth and a specific sparsity property (Wang et al., 2017). The modes are mostly compact around the central pulsation. VMD decomposes the original data $x(t)$ into the modes $s_{k}$ by formulating the constrained optimization problem as in Eq. 1 (Dragomiretskiy and Zosso, 2014).

$$
\begin{gathered}
\min _{\left\{s_{k}\right\},\left\{\omega_{k}\right\}}\left\{\sum_{k}\left\|\partial_{t}\left[\left(\delta(t)+\frac{j}{\pi t}\right) \times s_{k}(t)\right] e^{-j \omega_{k} t}\right\|_{2}^{2}\right\} \\
\text { Such that } \sum_{k} s_{k}(t)=x(t)
\end{gathered}
$$

Where $x(t)$ is the original time series data, $s_{k}$ is the decomposed modes, $k$ is the number of modes, $\omega_{k}$ is the centre frequency, $\delta$ is the Dirac distribution, $\otimes$ is the convolution operator.

The constrained minimization problem in Eq. 1 is transformed to the unconstrained optimization problem in Eq. 2 


$$
\begin{aligned}
L\left(\left\{y_{k}\right\},\left\{\omega_{k}\right\}, \lambda\right)= & \alpha \sum_{k}\left\|\partial_{t}\left[\left(\delta(t)+\frac{j}{\pi t}\right) \times y_{k}(t)\right] e^{-j \omega_{k} t}\right\|_{2}^{2} \\
& +\left\|y(t)-\sum_{k} y_{k}(t)\right\|_{2}^{2}+\left\langle\lambda(t), y(t)-\sum_{k} y_{k}(t)\right\rangle
\end{aligned}
$$

Where $a$ refers to the data fidelity balancing parameter for the penalty term. It encourages the reconstruction fidelity when noises are present in the data. We adopt ADMM (Alternate Direction Method of Multiplier) method to solve for the saddle point of the unconstrained problem in Eq. 2.

The VMD algorithm involves the following three steps. First, by applying Hilbert transform to the original data, the unilateral frequency spectrum of the original data is produced. Second, an exponential tuned to the centre frequency of the data is added to the unilateral frequency spectrum so that it is shifted and transformed to the baseband. Third, the mode for the bandwidth is estimated using $H^{1}$ Gaussian smoothness of the demodulated data.

Although the VMD model is useful in extracting data patterns in the multiscale domain, it infers data patterns based on historical data, rendering little power to predict future data movement. To resolve this issue, the QRNN model incorporates the neural network model into quantile regression modelling to estimate the quantile of data using past data observations as predictors. It allows more flexibility in modelling the complex nonlinear relationship. Unlike the linear assumption made in $\mathrm{QR}, \mathrm{QRNN}$ does not impose strict assumptions on the linear or nonlinear nature of the underlying relationship between the data quantile of the response variable and the predictor variables. It uses the neural network model to estimate the nonlinear relationship (Cannon, 2012; Koenker and Bassett, 1978).

Given the quantile $Q_{t}$ and the predictor variables $y_{t}$, QRNN model estimates the nonlinear relationship as in Eq. 3.

$$
Q_{t}(\tau)=f^{o}\left(\sum_{j=1}^{J} w_{j}^{o}(\tau) f^{h}\left(\sum_{i=1}^{p} w_{i j}^{h}(\tau) y_{t-i}+b^{h}(\tau)\right)+b^{o}(\tau)\right)
$$


where $b^{o}$ indicates the bias of the output layer, $w_{j}^{o}$ is the weight for the output layer, and $f^{o}(\cdot)$ is the transfer function in the output layer. $w_{i j}^{h}$ refers to the hidden layer weight, $f^{h}(\cdot)$ is the transfer function in the hidden layer, and $b^{h}(\tau)$ refers to the bias of the hidden layer.

However, there are two problems with the QRNN model. The first is that the solution of the model can be the local minimum instead of the global minimum in the feasible solution set. The model may fit the data with high in-sample estimation precision, but generalizations beyond the sample range are not guaranteed with the same level of precision and accuracy in the forecasting. Second, it is difficult to interpret and track the underlying dynamics, while allowing for flexibility in a nonparametric approach. Although it has the ability to model some unknown data dynamics in the crude oil market, the QRNN model offers very few insights into the underlying risk structure. In addition, its estimation accuracy is sensitive to the parameter choice, which is mainly selected using the trial and error method and the empirical data. It works as a good nonlinear approximation to risk features when modelling some known risk structure, but does not suffice when the underlying risk structure is unknown.

\subsection{Hypothesis development and VMD-QRNN model}

\subsubsection{Hypothesis development}

The patterns of crude oil price fluctuations change over time. These regimes are influenced and shaped by such different risk factors, notably as macroeconomic factors, government policy, and various market risks. The understanding of market risk in the crude oil market, however, is preliminary and studies on risk forecasting are rather limited and sparse. So far, studies on the crude oil risk estimates use econometric methods, such as the Generalized Autoregressive Conditional Heteroscedasticity (GARCH) model, among others, to model and forecast risk measures such as volatility and Value at Risk. These models assume uniform behaviour of risks in the market, i.e. risk in the crude oil market is generated from common risk factors, and these risk factors do not change over time. This notion is also supported by the dominant Efficient Market Hy- 
pothesis $(\mathrm{EMH})$, based on the assumption of homogeneity in the market, where liquidity is generated from noise traders in financial markets. However, it is widely accepted that crude oil is traded in a more heterogeneous market environment, where risk is represented by a distribution with a fat tail, stochastic volatility, and self-similarity (Barna et al., 2016). To address these issues, we develop a model, which takes into account both fundamental and transient risk factors associated with the crude oil market. Given the foregoing discussions, our hypothesis with regards to the heterogeneity in the crude oil markets is the following.

Hypothesis: In the crude oil market, two dominant factors determine the risk dynamics: the normal and transient risk factors, which can be distinguished by their multi-scale characteristics.

Theoretically, the finance literature has long classified events in financial markets into ordinary and extraordinary events (McDonald, 2013; Taleb, 2011). Recently, the Fractal Market Hypothesis [FMH] formalizes this classification by developing the theory whereby different major events in financial markets stem from investors decision making, based on a diverse range of criteria (Peters, 1994). Their distinct investment behaviours are distinguished by their individual beliefs, information constraints, and bounded rationality. When making investment decisions, Investors focus on different risk factors, such as fundamental factors versus technical analysis, and localized factors versus global factors. Among them, time horizon receives most research interest in the existing literature. Beyond the FMH, a group of researchers have proposed a more general Heterogeneous Market Hypothesis (HMH) to account for heterogeneous market features explicitly (Muller et al., 1993). Li et al. (2017) use the agent model to demonstrate that a limited time horizon exists in the market due to herding behaviour under the FMH. Rachev et al. (1999) and Weron and Weron (2000) show that the CED model can be used to approximate the global return distribution under the FMH and $\mathrm{HMH}$, respectively.

Although the FMH and HMH are theoretically sound for explaining the risk structure in the crude oil market, there is a lock of empirical methods 
and evidence for the to facilitate their implementation. More specifically, existing models are unable to identify and capture statistical characteristics of the multiscale risk structure, and thus, new models are developed using the interdisciplinary approach. Some empirical studies employ agent-based models, the Heterogeneous ARCH (HARCH) model and heterogeneous autoregressive model (HAR), and provide evidence that the joint influences of these risk factors on investors behaviour cause complex price changes and risk exposures, reinforced by characteristics such as self-similarity. For instance, by simulation, Li et al. (2014) and Li et al. (2017) use the agent-based model to show that interaction among agents is the source of market liquidity and can help ensure its stability. Mller et al. (1997) advocate that the HARCH model can capture volatilities over different time intervals, and suggest that the heterogeneous time horizon needs to be taken into account when analysing the market component in foreign exchange markets. Bianco et al. (2009) demonstrate the negative correlation between volatility forecasts and serial correlation (LeBaron effects) using the HAR model. Corsi (2009) proposes the HAR model based on HMH and demonstrates the effectiveness of HAR in reproducing the empirical data characteristics, such as long memory and fat tail, and improving forecasting performance. Cheong (2013) shows that the introduction of a non-homogeneous agent assumption in HAR, using the RV measure, improves the forecasting accuracy for the S\&P 500 stock market. Tao et al. (2018) propose a volatility forecasting model based on HAR, using the multi-fractal volatility measure, and find that this model produces more accurate forecasts. Qu and Ji (2016) demonstrate that higher forecasting accuracy can be achieved when the lag in HAR is dynamically selected. All these studies further confirm that heterogeneous dynamics are fundamental components of market structure.

To account for heterogeneous dynamics, we develop a model that has the ability to disaggregate the risk factors associated with the crude oil price fluctuation, into the normal and transient risk factors. The most conservative risk factor with the smallest number of exceedances is taken as the normal data component. After the normal risk factor is determined, we calculate the hybrid risk 
estimate using the normal risk factors and other decomposed factors. The scale, for which the most conservative hybrid risk estimate is produced, is referred to as the transient data component.

\subsubsection{Numerical algorithm}

Our proposed method requires three steps.

In the first step, our model deals with the separation of data components with distinct frequency bands by using the VMD model. We employ the VMD model to transform the original data into a pre-set number of data components at different scale in the multiscale domain. There have not, so far, been an established theory and methods on the selection of the decomposition scale. Studies in this field use some pre-set value for the maximum decomposition scale. For example, empirical studies conducted in Lahmiri (2016, 2015); He et al. (2018); Li et al. (2018); Mensi et al. (2017) use 10 as the decomposition scale. The pre-set number of data components is chosen based on this academic literature.

The uni-variate crude oil data $R_{t}=r_{1}, r_{2}, \ldots, r_{n}$ into the multiscale domain using VMD model as in Eq. 4.

$$
R(t)=\sum_{i=1}^{I} \operatorname{mode}_{i, t}
$$

Where mode $_{i, t}$ is the mode matrix. $I$ is the pre-set maximal level of decomposition scale.

In the second step, based on the in-sample data, we calculate the VaR for each decomposed modes using QRNN model. For scale $i$, we take the lagged modes with lag order $p$ as the input variables and the quantile of the crude oil prices as the response variables. The nonlinear relationship is modelled using the QRNN as in Eq. 5.

$$
V a R_{t, \tau, i}=f\left(\text { mode }_{t-1, i}, \text { mode }_{t-2, i}, \ldots, \text { mode }_{t-p, i} ; \theta_{\tau}\right) .
$$


Based on the calculated results, we identify the scale that contains both normal and transient factors of the original data among the decomposed modes. With respect to selecting the scale for the normal risk factors, we adopt VaR exceedances (VaR greater than the actual loss) as the criteria (Dowd, 2005). The normal factor stems from the fundamental factor and tends to be more stable, while the transient factor stems from the technical analysis and tends to be more volatile. The normal risk estimate tends to be more conservative compared to the transient factor (McDonald, 2013; Muller et al., 1993). We define the data series with the lowest VaR exceedances as the normal factor (the most conservative risk estimate). We select the scale with the lowest average exceedances as the normal factor. For selecting the scale for the transient factor, we calculate the exceedances of in-sample forecasts produced using the simple average ensemble of the chosen normal factors in the second step and one of the other extracted data components. We assume that there exist one normal and one transient factor, and therefore we select the scale with the lowest average exceedances as the transient scales among these factors.

In the third step, we produce forecasts using the out-of-sample data by the proposed model with the normal and transient factors identified in step 2 . We then evaluate the performance of the proposed model using the common performance measures, namely exceedances. VaR for Crude oil $V a R_{t, \tau}$ is supposed to be the linear combination of the VaR estimated under the normal market condition $\operatorname{VaR}_{n f, t, \tau}$ and VaR estimated under the transient market condition $V_{a} R_{t f, t, \tau}$ as in Eq. 6.

$$
V a R_{t, \tau}=(1-a) \times V \widehat{a R_{n f, t, \tau}}+a \times V \widehat{a R_{t f, t, \tau}}
$$

Where $a$ is the coefficient for the transient factor estimated using the multiscale model, Both $V \widehat{a R_{n f, t, \tau}}$ and $V \widehat{a R_{t f, t, \tau}}$ are forecasted using QRNN model with parameters estimated using decomposed data in the multiscale domains in step 2 .

The combination in the above equation is essentially an ensemble process. 
The ensemble models, often known as model averaging, combine the predictions from a set of individual models through either heterogeneous (i.e. individual models are of different types) or homogeneous (i.e. individual models are of the same type) ensemble methods (Moyano et al., 2018; Seijo-Pardo et al., 2017). The ensemble model has an ability to reduce the overfitting problem and improves the model generalization. The ensemble model proposes that each model bases its assumptions on the empirical data. They can capture only partial information in the data, as the empirical data taken from real data features violates the model's assumptions. Thus the ensemble forecasts are calculated using individual forecasts from independent models. This would reduce the variance of the forecast error of the ensemble member models forecasts and improve the forecasting accuracy of the ensemble forecasts. The ensemble model does not impose any restriction or assumptions on what types of models can be used to produce the individual forecasts. Therefore, they proposed that the ensembling process in step 3 can be generalized to the use of a wide range of models, such as typical econometric models and artificial intelligence models.

The parameter, $a$, can be estimated using a range of statistics and econometric techniques such as the linear regression model, the robust regression model, and the artificial intelligence model (Yu et al., 2005; He et al., 2018). If the parameters get larger, it implies that the influence of transient factors increases in strength, and vice versa. The model performance is sensitive to the choice of a. Abundant empirical evidence has shown the superior performance of the ensemble models such as Bates-Granger averaging and Bayesian model averaging (Wang et al., 2018a; Malone et al., 2014; Clemen, 1989). But no consensus has been reached on the optimal ensemble methods. Graefe et al. (2015) find that the performance of the simple average model is superior to Ensemble Bayesian Model Averaging (EBMA), in terms of not only forecasting performance, but also in the appeal of intuitive understanding and practical applications. They further argue that the average model is preferred, compared to more complex ensemble model in practice. In the practical situation, we expect that the weights for the ensemble model to be dynamic and it can be modelled using nonlinear 
models or artificial intelligence models in a data driven approach. However, these models introduce other model specification issues. For example, the estimated parameters may not be robust due to the data over-fitting issue and the violation of model assumptions when the model is estimated with different empirical datasets. The estimated model may not generalize well in the new data. Thus, in the simplest case, we adopt the averaging ensemble method in our model (Graefe et al., 2015). We average the VaR estimates for both normal and transient factors, i.e. a is 0.5 in Eq. 6.

In summary, both VMD and QRNN model are the integrated parts of our proposed model. They are combined to extract the appropriate risk structures to make VaR forecasts. The VMD model constructs a multiscale domain. The risk structures are identified and forecasts are made in the multiscale domain. The QRNN model estimates the individual VaR in a nonparametric manner, capturing the real risk changes in the market.

\section{Empirical analyses and discussion of the results}

\subsection{Experiment design}

We apply our proposed method to real crude oil data to evaluate the performance of our model. The real data that we employ for evaluation are the daily oil price in the US West Taxes Intermediate (WTI), UK Brent and OPEC market, which are the most liquid crude oil markets in the world. The data sources are the Energy Information Administration (EIA) of the US, and the Organization of the Petroleum Exporting Countries (OPEC). The data are downloaded from Quandl, which provides the centralized storage for a wide-range of datasets, including data from EIA and OPEC (Quandl, 2018). The experimental data for our evaluation ranges from the 2nd January 2003 to 4th January 2018. Our

final dataset consists of 3784 daily observations for the WTI market, 3817 daily observations for the Brent market, and 3874 daily observations for the OPEC market. We will then transform the original price data using the log difference method at the first order, so that the transformed data is detrended. 
The empirical dataset is divided into the training set and test set to facilitate the model estimation and model evaluation. The ratio for dataset division is determined based on the trial and error method, given the lack of a theoretical framework. In the literature, the ratio can range from $40 \%-60 \%$ to $80 \%-20 \%$ with sufficient number of observations reserved for the out-of-sample model evaluation, while 70\%-30\% is a popular choice (Dobbin and Simon, 2011; Lahmiri, 2016; Wang et al., 2018b; He et al., 2018, 2017). In our study, the in-sample data take up the first $70 \%$ of the dataset and are used to identify the scales that exhibit the transient data feature. The out-of-sample estimation takes up the remaining $30 \%$ of the dataset to calculate forecasting accuracy based on our proposed model. We assume a one-dollar portfolio asset.

Data analysis is conducted using Matlab 2013a computing software, while experiments with the models and algorithms are conducted using $\mathrm{R}$ statistical computing software. VMD models are implemented using vmd packages on R (Hamilton and Ferry, 2017). QRNN models are implemented using qrnn packages on R (Cannon, 2012, 2017).

\subsection{Experiment results}

The results of the in-sample data analyses are reported in Table 1.

Table 1: Descriptive statistics and statistical tests using the training dataset

\begin{tabular}{ccccccc}
\hline Market & Mean $\times 10^{-4}$ & Standard Deviation & Skewness & Kurtosis & $p_{J B}$ & $p_{B D S}$ \\
\hline WTI & 4.911 & 0.0269 & -0.0999 & 7.3634 & 0.001 & 0 \\
Brent & 5.5400 & 0.0242 & 0.0089 & 7.6655 & 0.001 & 0.0005 \\
OPEC & 5.0477 & 0.0179 & -0.3373 & 6.2702 & 0.001 & 0 \\
\hline
\end{tabular}

Notes: $p_{\mathrm{JB}}$ is the $p$-value for the Jarque-Bera (JB) statistic that tests the null hypothesis of Gaussian distribution, and $p_{\mathrm{BDS}}$ is the $p$-value for the BDS statistic that detects nonlinear serial dependence in returns. Table 1 presents descriptive statistics for returns in the WTI, Brent and OPEC

The experimental results show that the distribution of the three crude oil markets deviates from the normal distribution, which is consistent with prior 
studies. The kurtosis value is significantly larger than the standard value, 3 . The null hypothesis of both JB and BDS tests are rejected at the $5 \%$ level of significance. These statistics suggest that the crude oil data are not linearly dependent, and may contain unspecified nonlinear dynamics such as multiscale data features. Therefore, these nonlinear features in the crude oil market can be modelled using the VMD model and neural network approach in the QRNN model.

The decomposed modes are plotted in Figure 1.
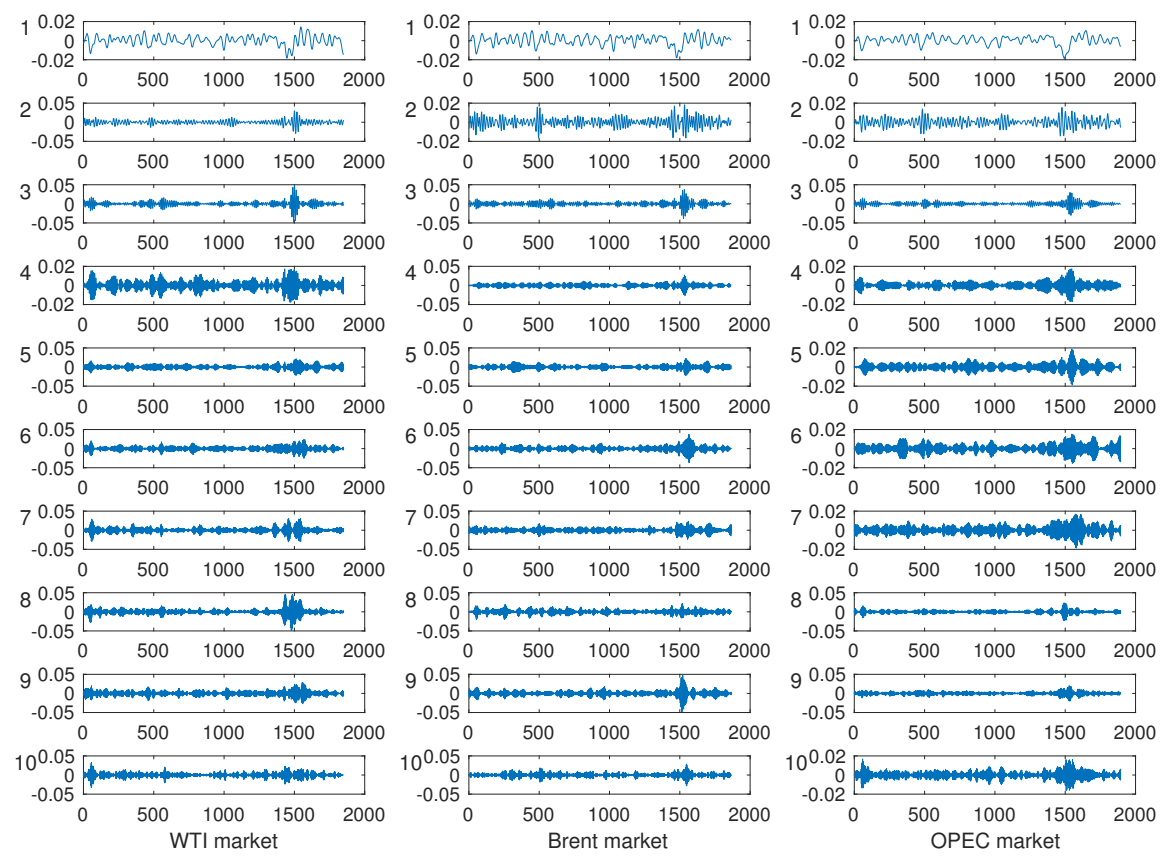

Figure 1: Plot of decomposed modes using the VMD model

Figure 1 shows that risk factors have distinct characteristics. Generally the volatilities of these risk factors increase as the scale goes up. The first scale exhibits the most stable behaviour and is most likely influenced by the marketwide normal risk factors such as macroeconomic factors and government policy, etc. The other scales exhibit more volatile behaviour and are more likely to be influenced by the transient risk factors than those in the first scale, i.e. 
the normal risk factors. Since the decomposed data components reconstruct the original crude oil price, these decomposed modes represent the candidate structure of risk factors in the crude oil price. The normal and transient risk factor in the hypothesis is expected to exist among these data components. However, there is no theory on the identification of the normal and transient factor in the literature. It is hard to tell which scale is more volatile than the other scales from direct visual inspection of the figure. We cannot identify these factors directly from the raw data and need to introduce the performance measure for risk estimate to provide quantitative evidence during the risk scale identification process.

We then forecast the VaR using the in-sample data for each of the scales. We follow the existing studies in the economic and finance literature to set the decomposition scales to 10 , and produce 10 different forecasts for 10 scales for each of the three markets (Lahmiri, 2016, 2015; He et al., 2018; Li et al., 2018; Mensi et al., 2017). The lag is determined by minimizing the information criteria such as Akaike Information Criteria (AIC) when ARMA-GARCH model is estimated with different lag choices. We have identified one lag for the WTI and OPEC markets and two lags for the Brent market. We, therefore, set the lag for the QRNN Model to 2 to include the maximum information, i.e. the QRNN model takes the returns of the current period over the two previous periods as the input. Although VaR can be estimated at any confidence level given, they are mostly estimated at three confidence levels, $95 \% 97$. 5\% and $99 \%$. We follow the existing literature and estimate it at the three confidence levels (Degiannakis and Potamia, 2017; Berger and Genay, 2018; Meng and Taylor, 2018). The number of exceedances for the forecasts at 10 scales are reported in Table 2 . 
Table 2: Exceedances results from VMD-QRNN model using in-sample data

\begin{tabular}{ccccccccccc}
\hline Market & $N_{1}$ & $N_{2}$ & $N_{3}$ & $N_{4}$ & $N_{5}$ & $N_{6}$ & $N_{7}$ & $N_{8}$ & $N_{9}$ & $N_{10}$ \\
\hline WTI $_{99 \%}$ & 3 & 0 & 2 & 8 & 5 & 6 & 5 & 10 & 5 & 2 \\
WTI $_{97.5 \%}$ & 14 & 14 & 13 & 17 & 12 & 17 & 16 & 21 & 17 & 14 \\
WTI $_{95 \%}$ & 25 & 26 & 30 & 29 & 21 & 31 & 33 & 32 & 32 & 28 \\
WTI $_{\text {Average }}$ & 14 & 13.3333 & 15 & 18 & $\mathbf{1 2 . 6 6 6 7}$ & 18 & 18 & 21 & 18 & 14.6667 \\
Brent $_{99 \%}$ & 2 & 5 & 7 & 4 & 5 & 9 & 10 & 4 & 2 & 4 \\
Brent $_{97.5 \%}$ & 8 & 13 & 17 & 13 & 15 & 19 & 17 & 12 & 10 & 13 \\
Brent $_{95 \%}$ & 25 & 28 & 28 & 29 & 22 & 30 & 28 & 25 & 27 & 19 \\
Brent $_{\text {Average }}$ & $\mathbf{1 1 . 6 6 6 7}$ & 15.3333 & 17.3333 & 15.3333 & 14 & 19.3333 & 18.3333 & 13.6667 & 13 & 12 \\
OPEC $_{99 \%}$ & 3 & 9 & 4 & 4 & 1 & 5 & 5 & 2 & 4 & 4 \\
OPEC $_{97.5 \%}$ & 11 & 21 & 13 & 10 & 8 & 15 & 13 & 13 & 11 & 14 \\
OPEC $_{95 \%}$ & 19 & 34 & 34 & 27 & 27 & 36 & 30 & 26 & 24 & 33 \\
OPEC $_{\text {Average }}$ & $\mathbf{1 1}$ & 21.3333 & 17 & 13.6667 & 12 & 18.6667 & 16 & 13.6667 & 13 & 17 \\
\hline
\end{tabular}

Notes: $N_{i}, i=1,2, \ldots, 10$ is the number of exceedances at the scale $i$ for different crude oil market at the confidence level $95 \%, 97.5 \%$ and $99 \%$, as well as the average value across all three confidence levels. Table 2 show the number of exceedances in the WTI, Brent and OPEC markets

Based on the experimental results in Table 2, the most conservative risk estimate among the risk estimates of 10 scales in each market is identified as the normal risk factor, that is, scale 5 for the WTI market and scale 1 for both the Brent and OPEC market, because VaR at theses scales has the lowest average number of exceedances.

Next, we conduct an exhaustive evaluation of the performance of our model across the ten different scales for use as the transient factors. The results are reported in Table 3 . 
Table 3: Exceedances results from VMD-QRNN model using the in-sample data

\begin{tabular}{ccccccccccc}
\hline Market & $N_{1}$ & $N_{2}$ & $N_{3}$ & $N_{4}$ & $N_{5}$ & $N_{6}$ & $N_{7}$ & $N_{8}$ & $N_{9}$ & $N_{10}$ \\
\hline $\mathbf{W T I}_{99 \%}$ & 4 & 2 & 5 & 6 & 5 & 5 & 5 & 7 & 4 & 4 \\
$\mathbf{W T I}_{97.5 \%}$ & 10 & 12 & 11 & 11 & 12 & 13 & 13 & 13 & 13 & 10 \\
$\mathbf{W T I}_{95 \%}$ & 22 & 21 & 23 & 23 & 21 & 26 & 22 & 24 & 24 & 22 \\
WTI $_{\text {Average }}$ & 12 & $\mathbf{1 1 . 6 6 6 7}$ & 13 & 13.3333 & 12.6667 & 14.6667 & 13.3333 & 14.6667 & 13.6667 & 12 \\
Brent $_{99 \%}$ & 2 & 2 & 2 & 1 & 2 & 2 & 2 & 1 & 1 & 1 \\
Brent $_{97.5 \%}$ & 8 & 10 & 8 & 10 & 7 & 10 & 11 & 9 & 7 & 11 \\
Brent $_{95 \%}$ & 25 & 22 & 21 & 20 & 22 & 23 & 22 & 21 & 22 & 18 \\
Brent $_{\text {Average }}$ & 11.6667 & 11.3333 & 10.3333 & 10.3333 & 10.3333 & 11.6667 & 11.6667 & 10.3333 & 10 & 10 \\
OPEC $_{99 \%}$ & 3 & 2 & 2 & 1 & 2 & 2 & 2 & 2 & 2 & 2 \\
OPEC $_{97.5 \%}$ & 11 & 13 & 6 & 6 & 7 & 13 & 8 & 9 & 8 & 11 \\
OPEC $_{95 \%}$ & 19 & 25 & 25 & 20 & 22 & 20 & 25 & 22 & 22 & 25 \\
OPEC $_{\text {Average }}$ & 11 & 13.3333 & 11 & $\mathbf{9}$ & 10.3333 & 11.6667 & 11.6667 & 11 & 10.6667 & 12.6667 \\
\hline Notes: $N_{i}, i=1,2, \ldots, 10$ & is the number of exceedances at the scale $i$ for different crude oil market at the confidence \\
level 95\%, 97.5\% and 99\%, as well as the average value across all three confidence levels. Table 3 presents the number \\
of exceedances in the WTI, Brent and OPEC markets
\end{tabular}

The results show that the scale that contains the transient factor has been identified as scale 2 for the WTI, scale 10 for the Brent market, and scale 4 for the OPEC market. These scales produce the most conservative risk estimate for the respective market, when they are chosen to be the transient factor. We demonstrate that both normal risk factors and transient risk factors contribute to the overall risk fluctuations. These results are consistent with our hypothesis that the normal risk factors provide the baseline estimate. The transient risk factors provide the estimate for the risk over and above the normal risk level under the transient and extreme market condition. They are both critical to measuring the market risk level accurately. Our results demonstrate that scale 1 , representing the normal risk factors, has a lower number of exceedances than the transient risk factors. The normal risk factors have larger value and are more conservative. ${ }^{1}$ In the meantime, although the results reported in Table 3

\footnotetext{
${ }^{1}$ It is worth noting that we have run the experiment several times. The number of exceedances for VaR estimated may change slightly for each experiment, but the scale selected
} 
show the same number of exceedances for scale 9 and 10 in the Brent market, when the neural network is initialized with different sets of weights generated randomly, the number of exceedances for scale 9 and 10 actually differs and the number of exceedances for scale 10 is the smallest. Thus we choose scale 10 for the Brent market.

Next, we forecast the VaR using the out-of-sample data by three benchmark models, namely the ARMA-GARCH, QRNN and VMD-QRNN models, and to evaluate and compare their performance to our proposed model. The performance measures are reported in Table 4.

Table 4: Forecasting performance from different models using the out-of-sample data

\begin{tabular}{cccccccccc}
\hline Market & $N_{95 \%}$ & $p_{95 \%}$ & $M S E_{95 \%}$ & $N_{97.5 \%}$ & $p_{97.5 \%}$ & $M S E_{97.5 \%}$ & $N_{99 \%}$ & $p_{99 \%}$ & $M S E_{99 \%}$ \\
\hline ARMA-GARCH $_{W T I}$ & 52 & 0.5120 & 0.0020 & 33 & 0.3912 & 0.0026 & 20 & 0.0198 & 0.0034 \\
QRNN $_{W T I}$ & 74 & 0.0245 & 0.0021 & 49 & 0.0004 & 0.0029 & 19 & 0.0374 & 0.0043 \\
VMD-QRNN $_{W T I}$ & 71 & 0.0613 & 0.0020 & 34 & 0.2995 & 0.0032 & 15 & 0.2994 & 0.0044 \\
ARMA-GARCH $_{\text {Brent }}$ & 69 & 0.1222 & 0.0016 & 35 & 0.2433 & 0.0021 & 17 & 0.1241 & 0.0028 \\
QRNN $_{\text {Brent }}$ & 56 & 0.8649 & 0.0021 & 39 & 0.0625 & 0.0027 & 26 & 0.0002 & 0.0037 \\
VMD-QRNN $_{\text {Brent }}$ & 57 & 0.9729 & 0.0018 & 29 & 0.9435 & 0.0022 & 16 & 0.2023 & 0.0033 \\
ARMA-GARCH $_{O P E C}$ & 63 & 0.5150 & 0.0011 & 33 & 0.4674 & 0.0015 & 18 & 0.0817 & 0.0019 \\
QRNN $_{O P E C}$ & 80 & 0.0052 & 0.0013 & 50 & 0.0003 & 0.0018 & 30 & 0 & 0.0027 \\
VMD-QRNN $_{O P E C}$ & 56 & 0.7762 & 0.0011 & 38 & 0.1081 & 0.0015 & 10 & 0.6246 & 0.0025 \\
\hline
\end{tabular}

Notes: $N_{c l}, c l=95 \%, 97.5 \%, 99 \%$ is the number of exceedances at confidence level $c l . P_{c l}$ is the p -value for the likelihood ratio test of unconditional coverage. $M S E_{c l}$ is the Mean Square Error at confidence level $c l$.

The experimental results in Table 4 show that, in general, our proposed model has passed the unconditional coverage test for all the three markets at all the confidence levels. By comparison, the QRNN model fails to pass the test for the WTI and OPEC market at all the confidence levels and for the Brent market at the $99 \%$ and $97.5 \%$ confidence levels. Although ARMA-GARCH passes the unconditional coverage test for the WTI and OPEC market at both $95 \%$ and $97.5 \%$ confidence levels respectively, it fails to pass the test for the

remain the same. 
WTI market at the $99 \%$ confidence level. It passes the test for the Brent market at $95 \%, 97.5 \%$ and $99 \%$ confidence levels and OPEC market at the $99 \%$ confidence level. Overall, the evaluation results demonstrate that our proposed model provides a more reliable and robust risk estimate, with increased forecasting accuracy, than the benchmark ARMA-GARCH model and QRNN model. Most significantly, our proposed model does not require either conservative or aggressive risk preference, and the risk estimate produced by our model tracks the downside risk movement. Compared to the traditional risk models such as the ARMA-GARCH model, our proposed model produces more conservative estimates when the risk is underestimated, and vice versa.

These significant results confirm our hypothesis: a diverse range of risk factors prevails in the crude oil market. They can be divided into two principal groups: normal and transient type risks. Each type of risk factor has unique behaviours and patterns. In our experiment, this is reflected by the difference between the model parameters when estimated by applying normal and transient risk factors. Our results also demonstrate that both normal and transient factors need to be identified based on model performance and quantitative performance measure, as in this paper. The results may contradict the intuitive selection based on the visual inspection. In this paper, when we examine Figure 1, data series at scale 1 present the most stable pattern, and hence are chosen as the normal risk factor among three crude oil markets. However, our empirical analysis in Table 2 suggests that scale 5 is the normal risk factor for the WTI market and scale 1 is the normal risk factor for the Brent and OPEC markets. In the meantime, there does not seem to be clear pattern for the determination of the scale for the transient risk factor, as our empirical analysis has identified a different scale for both normal and transient risk factors for the WTI, Brent and OPEC markets.

Our model is robust in that it involves no optimization procedure and has been tested on an extensive dataset across three main crude oil markets over a considerable length of time. The multiscale model proposed for crude oil risk forecasting in this paper can be generalized to the risk forecasting in other 
economic and financial markets. Ultimately, it represents a general multiscale method that improves the modelling accuracy when modeling the data with multiscale characteristics in other markets (He et al., 2009; Bao et al., 2018; Pradeepkumar and Ravi, 2017).

Furthermore, in respect of determining the scale for normal and transient factors, the simplest minimum average exceedances criteria that we have introduced into our model can be easily relaxed and extended. The significant development in our proposed method also indicates that the criteria can be refined by taking into account the heterogeneous nature of crude oil data so as to produce more accurate risk estimates by applying more realistic assumptions. For example, multiple normal factors and transient factors may be modelled and estimated simultaneously.

As the normal risk factors refer to the macroeconomic factors, they are expected to be less volatile and more long-term orientated. Empirical evidence shows the existence of correlation and the spillover effect between the macroeconomic factors and the crude oil movement in the long run equilibrium condition. For example, Mensah et al. (2017) demonstrate the long-term equilibrium relationship between oil price and major exchange rates by means of a cointegration model. Mei-Se et al. (2018) show the long run relationship among metal prices, oil prices and exchange rates. Yang et al. (2018) find the long run correlation between oil prices and exchange rates based on Dynamic Conditional Correlation - Mixed Data Sampling mode.

As the transient risk factors refer to the influence of the unexpected shocks, they are supposed to be more volatile and demonstrate drastically different characteristics. Empirical studies have demonstrated the transient characteristics such as jumps in the short-term time horizon. For example, Li et al. (2017) find the jump spillover effect between oil prices and exchange rates by applying the stochastic volatility model. Jawadi et al. (2016) find the volatility spillover through jumps from the exchange rate to oil price. 


\section{Conclusion}

In this paper, we have proposed a new Value at Risk estimate model based on the VMD and QRNN models. We demonstrate how our approach has the power to separate the data components with normal and transient characteristics, which leads to a superior, more efficient model fit and the capacity to obtain greater precision in crude oil pricing. We identify the transient factor that contributes most to the crude oil price fluctuations.

In order to verify the effectiveness of our proposed pricing model, we apply the real data of the major crude oil markets and compare the performance of our model against the benchmark, i.e. Quantile Regression Neural Network model and ARMA-GARCH model. The evaluation results consistently demonstrate that our model can generate parameters for crude oil prices that are superior to the existing benchmark approaches such as ARMA-GARCH and QRNN model, with an improved risk estimation performance. Our approach is of economic significance since our model demonstrates that different levels of risk affect the parameter estimates of complex crude oil data. Hence, modelling data by taking account of differential levels of risk will lead to a better model fit.

Most significantly, our study provides clear evidence that crude oil data contains a multiscale risk structure. These risk factors incorporate different features that may interact with each other to form the complex nonlinear dynamics in the risk behaviour of the crude oil price. Practically, the proposed model can serve as a more flexible enterprise's risk management system. The risk management decision can be made based on different risk preferences and market conditions. Under more extreme and turbulent market conditions, the transient risk factors take precedence and can be singled out for more accurate risk analysis. The weights for normal and transient risk estimates can be adjusted flexibly according to the risk preference of different types of investors.

Our findings will intensify interest in new models for energy risk forecasting and stimulate subsequent research for energy risk management research. Our technique will facilitate more accurate measurement of downside risk to 
inform the energy financing decision. Work in this paper has methodological and empirical value by revealing the existence of different types of risk factors in the energy markets, and the construction of the new multi scale methodology to capture most important risk factors and forecast the downside risk more reliably. Future investigation can focus on the more accurate risk forecasting model with the increased relaxation of the assumptions of the normal and transient risk factors, such as their number and time series characteristics. Further studies are recommended to consider the development of a suitable and robust ensemble algorithm for integrating normal and transient energy risk factors in constructing new risk forecasting models, and the follow-up analysis of the unique characteristics of both risk factors in the energy risk analysis.

\section{Acknowledgments}

This work is supported by the National Natural Science Foundation of China (NSFC No. 71671013), and Humanities and Social Sciences Youth Foundation of Ministry of Education of China (No. 16YJC790026)

\section{References}

Balcilar, M., Ozdemir, Z. A., Yetkiner, H., 2014. Are there really bubbles in oil prices? Physica A: Statistical Mechanics and its Applications 416, 631 - 638.

Bao, C., Li, J., Wu, D., 2018. A fuzzy mapping framework for risk aggregation based on risk matrices. Journal of Risk Research 21 (5), 539-561.

Barna, F., Dima, S. M., Dima, B., Pasca, L., 2016. Fractal Market Hypothesis: the Emergent Financial Market Case. Economic Computation and Economic Cybernetics Studies and Research 50 (2), 137-150.

Berger, T., Genay, R., 2018. Improving daily Value-at-Risk forecasts: The relevance of short-run volatility for regulatory quality assessment. Journal of Economic Dynamics and Control 92, $30-46$. 
Bianco, S., Corsi, F., Reno, R., 2009. Intraday LeBaron effects. Proceedings of the National Academy of Sciences of the United States of America 106 (28), 11439-11443.

Cannon, A. J., 2012. Quantile regression neural networks: Implementation in R and application to precipitation downscaling (vol 37, pg 1277, 2011). Computers $\&$ Geosciences 46, 9-9.

Cannon, A. J., 2017. Non-crossing nonlinear regression quantiles by monotone composite quantile regression neural network, with application to rainfall extremes. EarthArXiv ¡https://eartharxiv.org/wg7sni, doi:10.17605/OSF.IO/WG7SN.

Cheong, C. W., 2013. The Computational of Stock Market Volatility From the Perspective of Hetergeneous Market Hypothesis. Economic Computation and Economic Cybernetics Studies and Research 47 (2), 247-260.

Clemen, R. T., 1989. Combining forecasts: A review and annotated bibliography. International Journal of Forecasting 5 (4), 559 - 583.

Corsi, F., 2009. A Simple Approximate Long-Memory Model of Realized Volatility. Journal of Financial Econometrics 7 (2), 174-196.

Degiannakis, S., Potamia, A., 2017. Multiple-days-ahead value-at-risk and expected shortfall forecasting for stock indices, commodities and exchange rates: Inter-day versus intra-day data. International Review of Financial Analysis $49,176-190$.

Dobbin, K. K., Simon, R. M., 2011. Optimally splitting cases for training and testing high dimensional classifiers. BMC Medical Genomics 4 (1), 31.

Dowd, K., 2005. Measuring Market Risk. John Wiley \& Sons Inc.

Dragomiretskiy, K., Zosso, D., 2014. Variational Mode Decomposition. Ieee Transactions on Signal Processing 62 (3), 531-544. 
Engle, R. F., Manganelli, S., 2004. CAViaR: Conditional autoregressive value at risk by regression quantiles. Journal of Business 85 Economic Statistics $22(4), 367-381$.

Fama, E., 1965. The Behavior of Stock Market Prices. Journal of Business 38 (1), 34-105.

Fama, E. F., 1970. Efficient Capital Markets: A Review of Theory and Empirical Work. The Journal of Finance 25 (2), 383-417.

Graefe, A., Kchenhoff, H., Stierle, V., Riedl, B., 2015. Limitations of Ensemble Bayesian Model Averaging for forecasting social science problems. International Journal of Forecasting 31 (3), 943 - 951.

Hamilton, N., Ferry, M., 2017. vmd: Variational Mode Decomposition.

He, K., Chen, Y., Tso, G. K., 2017. Price forecasting in the precious metal market: A multivariate EMD denoising approach. Resources Policy 54, 9 24.

He, K., Chen, Y., Tso, G. K., 2018. Forecasting exchange rate using Variational Mode Decomposition and entropy theory. Physica A: Statistical Mechanics and its Applications 510, $15-25$.

He, K. J., Xie, C., Chen, S., Lai, K. K., 2009. Estimating VaR in crude oil market: A novel multi-scale non-linear ensemble approach incorporating wavelet analysis and neural network. Neurocomputing 72 (16 - 18), 3428 - 3438.

Herrera, A. M., Hu, L., Pastor, D., 2018. Forecasting crude oil price volatility. International Journal of Forecasting 34 (4), 622 - 635.

Hosseini, S. A., Shah, N., 2009. Multiscale modelling of hydrothermal biomass pretreatment for chip size optimization. Bioresource Technology 100 (9), 2621 -2628 .

Huang, N. E., Shen, Z., Long, S. R., Wu, M. C., Shih, H. H., Zheng, Q., Yen, N.-C., Tung, C. C., Liu, H. H., 1998. The empirical mode decomposition and 
the Hilbert spectrum for nonlinear and non-stationary time series analysis. Proceedings of the Royal Society of London A: Mathematical, Physical and Engineering Sciences 454 (1971), 903-995.

Huang, S., An, H., Wen, S., An, F., 2017. Revisiting driving factors of oil price shocks across time scales. Energy 139, 617-629.

Jawadi, F., Louhichi, W., Ameur, H. B., Cheffou, A. I., 2016. On oil-US exchange rate volatility relationships: An intraday analysis. Economic Modelling $59,329-334$.

Ji, Q., Bouri, E., Roubaud, D., Shahzad, S. J. H., 2018d. Risk spillover between energy and agricultural commodity markets: A dependence-switching CoVaR-copula model. Energy Economics 75, 14- 27.

Ji, Q., Geng, J.-B., Tiwari, A. K., 2018. Information spillovers and connectedness networks in the oil and gas markets. Energy Economics 75, $71-84$.

Ji, Q., Liu, B.-Y., Fan, Y., 2018c. Risk dependence of CoVaR and structural change between oil prices and exchange rates: A time-varying copula model. Energy Economics.

Ji, Q., Zhang, D., 2018. Chinas crude oil futures: Introduction and some stylized facts. Finance Research Letters.

Ji, Q., Zhang, D., bo Geng, J., 2018. Information linkage, dynamic spillovers in prices and volatility between the carbon and energy markets. Journal of Cleaner Production 198, $972-978$.

Jianwei, E., Bao, Y. L., Ye, J. M., 2017. Crude oil price analysis and forecasting based on variational mode decomposition and independent component analysis. Physica A-statistical Mechanics and Its Applications 484, 412-427.

Koenker, R., Bassett, G., 1978. Regression Quantiles. Econometrica 46 (1), 3350. 
Lahmiri, S., 2015. Long memory in international financial markets trends and short movements during 2008 financial crisis based on variational mode decomposition and detrended fluctuation analysis. Physica A-statistical Mechanics and Its Applications 437, 130-138.

Lahmiri, S., 2016. Intraday stock price forecasting based on variational mode decomposition. Journal of Computational Science 12, 23-27.

Li, D., Li, R., Sun, Q., 2017. How the heterogeneity in investment horizons affects market trends. Applied Economics 49 (15), 1473-1482.

Li, D.-Y., Nishimura, Y., Men, M., 2014. Fractal markets: Liquidity and investors on different time horizons. Physica A: Statistical Mechanics and its Applications 407, 144-151.

Li, J., Yao, X., Sun, X., Wu, D., 2018. Determining the fuzzy measures in multiple criteria decision aiding from the tolerance perspective. European Journal of Operational Research 264 (2), 428 - 439.

Li, X.-P., Zhou, C.-Y., Wu, C.-F., 2017. Jump spillover between oil prices and exchange rates. Physica A: Statistical Mechanics and its Applications 486, 656 -667 .

Liu, T.-Y., Lee, C.-C., 2018. Will the energy price bubble burst? Energy 150, $276-288$.

Malone, B. P., Minasny, B., Odgers, N. P., McBratney, A. B., 2014. Using model averaging to combine soil property rasters from legacy soil maps and from point data. Geoderma 232-234, 34-44.

Mandelbrot, B., 1963. New Methods in Statistical Economics. Journal of Political Economy 71 (5), 421-440.

McDonald, R., 2013. Derivatives Markets. Pearson series in finance. Pearson. 
Mei-Se, C., Shu-Jung, C. L., Chien-Chiang, L., 2018. Time-varying comovement of the prices of three metals and oil: Evidence from recursive cointegration. Resources Policy 57, 186 - 195.

Meng, X., Taylor, J. W., 2018. An approximate long-memory range-based approach for value at risk estimation. International Journal of Forecasting 34 (3), $377-388$.

Mensah, L., Obi, P., Bokpin, G., 2017. Cointegration test of oil price and us dollar exchange rates for some oil dependent economies. Research in International Business and Finance 42, $304-311$.

Mensi, W., Hammoudeh, S., Shahzad, S. J. H., Shahbaz, M., 2017. Modeling systemic risk and dependence structure between oil and stock markets using a variational mode decomposition-based copula method. Journal of Banking 83 Finance 75, 258-279.

Miao, H., Ramchander, S., Wang, T., Yang, D., 2017. Influential factors in crude oil price forecasting. Energy Economics 68, $77-88$.

Mohammadi, H., Su, L., 2010. International evidence on crude oil price dynamics: Applications of ARIMA-GARCH models. Energy Economics 32 (5), 1001 $-1008$.

Moyano, J. M., Gibaja, E. L., Cios, K. J., Ventura, S., 2018. Review of ensembles of multi-label classifiers: Models, experimental study and prospects. Information Fusion 44, 33 - 45.

Muller, U. A., Dacorogna, M., Dave, R. D., Pictet, O. V., Olsen, R., Ward, J., 1993. Fractals and Intrinsic Time - a Challenge to Econometricians. Working Papers 1993-08-16, Olsen and Associates.

Mller, U. A., Dacorogna, M. M., Dav, R. D., Olsen, R. B., Pictet, O. V., von Weizscker, J. E., 1997. Volatilities of different time resolutions analysing the dynamics of market components. Journal of Empirical Finance 4 (2), 213 239. 
Narayan, P. K., 2017. Energy Finance: An Overview. Energy Economics 66, 535.

Peters, E., 1994. Fractal Market Analysis: Applying Chaos Theory to Investment and Economics. Wiley Finance. Wiley.

Plourde, A., Watkins, G., 1998. Crude Oil Prices Between 1985 and 1994: How Volatile in Relation to Other Commodities? Resource and Energy Economics $20(3), 245-262$.

Pradeepkumar, D., Ravi, V., 2017. Forecasting financial time series volatility using Particle Swarm Optimization trained Quantile Regression Neural Network. Applied Soft Computing 58, $35-52$.

Qiu, X., Ren, Y., Suganthan, P. N., Amaratunga, G. A., 2017. Empirical Mode Decomposition based ensemble deep learning for load demand time series forecasting. Applied Soft Computing 54, $246-255$.

Qu, H., Ji, P., 2016. Modeling Realized Volatility Dynamics with a Genetic Algorithm. Journal of Forecasting 35 (5), 434-444.

Quandl, 2018. EIA and OPEC Various End-Of-Day Data.

Rachev, S., Weron, A., Weron, R., 1999. CED model for asset returns and fractal market hypothesis. Mathematical and Computer Modelling 29 (10-12), $23-36$.

Sadeghi, M., Shavvalpour, S., 2006. Energy risk management and value at risk modeling. Energy Policy 34 (18), 3367 - 3373.

Seijo-Pardo, B., Porto-Daz, I., Boln-Canedo, V., Alonso-Betanzos, A., 2017. Ensemble feature selection: Homogeneous and heterogeneous approaches. Knowledge-Based Systems 118, $124-139$.

Su, C.-W., Li, Z.-Z., Chang, H.-L., Lobon, O.-R., 2017. When Will Occur the Crude Oil Bubbles? Energy Policy 102, 1 - 6. 
Taleb, N., 2011. The Black Swan: The Impact of the Highly Improbable. Allen Lane.

Tao, Q., Wei, Y., Liu, J., Zhang, T., 2018. Modeling and forecasting multifractal volatility established upon the heterogeneous market hypothesis. International Review of Economics 85 Finance 54, 143-153.

Taylor, J. W., 2010. A quantile regression neural network approach to estimating the conditional density of multiperiod returns. Journal of Forecasting.

Wang, D., Luo, H., Grunder, O., Lin, Y., 2017. Multi-step ahead wind speed forecasting using an improved wavelet neural network combining variational mode decomposition and phase space reconstruction. Renewable Energy 113, $1345-1358$.

Wang, J., Athanasopoulos, G., Hyndman, R. J., Wang, S., 2018. Crude oil price forecasting based on internet concern using an extreme learning machine. International Journal of Forecasting 34 (4), 665 - 677.

Wang, J., Li, X., Hong, T., Wang, S., 2018. A semi-heterogeneous approach to combining crude oil price forecasts. Information Sciences 460-461, 279 - 292.

Wang, J., Wang, J., 2017. Forecasting stochastic neural network based on financial empirical mode decomposition. Neural Networks 90, 8 - 20.

Wang, Y., Wu, C., Wei, Y., 2011. Can GARCH-class models capture long memory in WTI crude oil markets? Economic Modelling 28 (3), 921 - 927.

Weron, A., Weron, R., 2000. Fractal market hypothesis and two power-laws. Chaos Solitions 83 Fractals 11 (1-3), 289-296.

Xu, Q. F., Liu, X., Jiang, C. X., Yu, K. M., 2016. Quantile autoregression neural network model with applications to evaluating value at risk. Applied Soft Computing 49, 1 - 12 . 
Yang, L., Cai, X. J., Hamori, S., 2018. What determines the long-term correlation between oil prices and exchange rates? The North American Journal of Economics and Finance 44, 140 - 152.

Yang, Z., Ce, L., Lian, L., 2017. Electricity price forecasting by a hybrid model, combining wavelet transform, ARMA and kernel-based extreme learning machine methods. Applied Energy 190, 291-305.

Yu, L., Wang, S., Lai, K., 2005. A novel nonlinear ensemble forecasting model incorporating GLAR and ANN for foreign exchange rates. Computers $\& 3$ Operations Research 32 (10), 2523 - 2541.

Zhang, D., 2017. Oil shocks and stock markets revisited: Measuring connectedness from a global perspective. Energy Economics 62, 323 - 333.

Zhang, D., 2018. Energy Finance: Background, Concept, and Recent Developments. Emerging Markets Finance and Trade 54 (8), 1687-1692.

Zhang, D., Broadstock, D. C., 2018. Global financial crisis and rising connectedness in the international commodity markets. International Review of Financial Analysis.

Zhang, Y., Li, C., Li, a., 2018. Wavelet transform and Kernel-based extreme learning machine for electricity price forecasting. Energy Systems - Optimization Modeling Simulation and Economic Aspects 9 (1), 113-134.

Zhu, B. Z., Han, D., Wang, P., Wu, Z. C., Zhang, T., Wei, Y. M., 2017. Forecasting carbon price using empirical mode decomposition and evolutionary least squares support vector regression. Applied Energy 191, 521-530. 